Artículo de reflexión E16A11. * Recibido: 10.12.2019. * Aprobado versión final: 28.12.2019. JEL: O.598; O2.86; O2.123; H.309. Pp.21-28. doi: 10.33571/teuken.v11n16a1

\title{
Políticas públicas de ciencia y tecnología en América Latina. Ante la encrucijada de los cambios políticos.
}

\author{
Public policies of science and technology in Latin America. \\ At the crossroads of political changes.
}

\author{
Rosalba Casas \\ MÉXICO
}

\begin{abstract}
Resumen: La orientación de las políticas de ciencia y tecnología en América Latina ha estado influenciada por múltiples factores, como los relacionados con las estrategias de desarrollo que se han implementado en los diferentes momentos de cambios políticos. La motivación de esta reflexión es discutir cómo estas políticas se han delineado y cómo la generación y aplicación de la ciencia y la tecnología debería incidir en nuestras sociedades. Se reflexiona sobre el avance en la concepción de una estrategia alternativa para integrar la ciencia y tecnología al desarrollo social sostenible y sustentable de América Latina.
\end{abstract}

Palabras clave: políticas públicas de ciencia y tecnología; cambios políticos; ciencia y tecnología en latam; estudios filosóficos y sociales sobre ciencia y tecnología.

Abstract: The orientation of science and technology policies in Latin American has been influenced by multiple factors, such as related to development strategies, which have been implemented at different moments of political change. The motivation of this reflection is to discuss how these policies have been outlined, and how the generation and application of science and technology should affect our societies; this manuscript draws attention to the progress in the conception of an alternative strategy to integrate science and technology to the sustainable social development of Latin America.

Keywords: science and technology public policies; political changes; science and technology in latam; philosophical and social studies on science and technology.

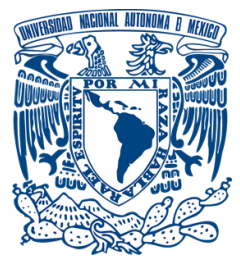

Rosalba Casas es Socióloga de la Universidad Nacional Autónoma de México; Maestra en Historia y Sociopolítica de la Ciencia de la Universidad de Montreal, Canadá, y Doctora en Política Científica y Tecnológica de la Universidad de Sussex, Inglaterra. Investigadora Titular C de Tiempo Completo del Instituto de Investigaciones Sociales de la Universidad Nacional Autónoma de México, Investigadora Nacional Nivel III del SNI en el Área de las Ciencias Sociales y PRIDE D, y Miembro de la Academia Mexicana de Ciencias. 


\title{
Políticas públicas de ciência e tecnologia na América Latina.
}

Na encruzilhada de mudanças políticas

\begin{abstract}
Resumo: A orientação das políticas de ciência e tecnologia na latinoamericana foi influenciada por múltiplos fatores, como os relacionados a estratégias de desenvolvimento, implementadas em diferentes momentos de mudança política. A motivação deste artigo é discutir como essas políticas foram delineadas e como a geração e aplicação de ciência e tecnologia devem afetar nossas sociedades; este manuscrito reflete sobre o progresso na concepção de uma estratégia alternativa para integrar ciência e tecnologia ao desenvolvimento social sustentável e sustentável da América Latina.
\end{abstract}

Palavras-chave: Políticas públicas de ciência e tecnologia; mudanças políticas; ciência e tecnologia na latam; estudos filosóficos e sociais sobre ciência e tecnologia.

\section{Introducción}

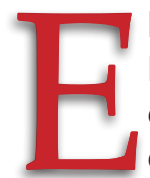

I desarrollo de las políticas de ciencia y tecnología en América Latina ha estado influido por numerosos factores, como las estrategias de desarrollo de nuestros países, las orientaciones de esas políticas en el marco internacional, las recomendaciones de los organismos internacionales y las concepciones sobre el papel de esas actividades para nuestras sociedades. Las políticas han sido un ámbito sensible a la voluntad política de los gobiernos, lo que revela la escasa comprensión que ha tenido el Estado en nuestros países sobre su importancia para el desarrollo y como factor para contribuir a mejorar las condiciones de vida de la población. La alternancia de los gobiernos y los cambios políticos resultantes, si bien han generado modificaciones de matiz en el discurso político, también han incidido en una construcción limitada de una nueva concepción que defina orientaciones que se plasmen en políticas coherentes y consistentes de ciencia y tecnología para la inclusión social.

De esto hemos dado cuenta en trabajos previos (Casas, Corona y Rivera, 2014) en los que difundimos resultados sobre los cambios en las políticas de ciencia y tecnología, a través del análisis de los discursos oficiales y de los planes y programas nacionales para el desarrollo científico, tecnológico y de innovación en países latinoamericanos, que durante el presente siglo dieron un giro hacia gobiernos progresistas y los comparamos con lo que ocurría en regímenes políticos y económicos claramente neoliberales. El discurso político de distintos gobiernos de la región ha enfatizado en el papel de la ciencia y la tecnología para 
objetivos de inclusión social, como los que actualmente se manifiestan en México, ${ }^{1}$ y que condujo a diversos países como Argentina, Brasil, Uruguay y Colombia a la introducción de instrumentos de política, que han tenido como propósito atender problemas sociales prioritarios (Arancibia, 2019); no obstante, la realidad es que se ha avanzado poco en la concepción de una estrategia alternativa para integrar la ciencia y tecnología al desarrollo social sostenible y sustentable.

Considerando las dificultades que se enfrentan desde las perspectivas teórica y práctica para establecer una relación directa entre ciencia, tecnología y sociedad y poniendo el énfasis en algunos cambios incipientes, se puede afirmar que, en los últimos veinte años, hemos avanzando en la región hacia una concepción de ciencia y tecnología como un medio y no como un fin en sí mismas, como ha sido la perspectiva dominante por tanto tiempo. Los planteamientos de Políticas de Ciencia, Tecnología e Innovación (PCTI) contenidos en planes y programas en distintos países latinoamericanos coinciden cada vez más en que la ciencia y la tecnología son recursos fundamentales para el logro de objetivos de desarrollo sostenible y de inclusión social. Así mismo, lo que hemos observado en términos generales (Casas, Corona y Rivera, 2014) es que en diversos países se ha intentado operar un cambio en la manera de entender el desarrollo de la ciencia, la tecnología, la innovación y sus interacciones. Se ha ido transitando lentamente de un discurso en el que predominaban las iniciativas e instrumentos basados en el enfoque lineal, a un discurso en el que se da mayor relevancia al enfoque sistémico, a la importancia de la interacción entre diferentes agentes y al concurso coordinado de múltiples actores y disciplinas cuando de problemas sociales complejos se trata.

Sin embargo, esta concepción dista mucho de ser la dominante al interior de las comunidades académicas, dentro de las que se encuentran grupos y élites que han ejercido un papel preponderante en nuestros países en las orientaciones de esas actividades sustentadas en una idea lineal, ofertista, que no incorpora aspectos como la responsabilidad y la

1 El criterio editorial de nuestra publicación con respecto a las palabras transliteradas del náhuatl se resume en que utilizamos la forma fonética del español, pues en esta lengua el grafema $X$ tiene un sonido único [ks] y no se pronuncia como J. En el uso cultural mejicano tampoco hay uniformidad con respecto a su pronunciación pues en algunas palabras de origen náhuatl la $X$ se pronuncia como tal y en otras se pronuncia como J. La Real Academia Española de la Lengua y la Academia Colombiana de la Lengua aceptan el uso de ambas formas de escritura. En este caso, nuestra autora invitada ha pedido expresamente que en su artículo utilicemos la forma transliterada de la palabra escrita con Xy en consecuencia hemos aceptado su solicitud. 
relevancia social. En virtud de que las PCTI en América Latina y el Caribe se han realizado durante mucho tiempo siguiendo el modelo lineal, el sector académico se ha convertido en un actor central tanto en el diseño, ejecución y evaluación de las políticas y, en ese sentido, tienden a reflejar mucho más las aspiraciones y los objetivos del sector académico y menos las intenciones del hacedor de políticas del gobierno o de la sociedad. El sector académico, principalmente el público, es el contexto social en el que se concentra la generación de conocimiento en nuestros países, en el que la posición sobre el papel de la ciencia y la tecnología ha estado determinada por los contextos y orientaciones institucionales y por los valores que han regido a la ciencia y la tecnología.

Retomando lo argumentado por Velho (2011:119), en el sentido de que es el concepto dominante de ciencia el que moldea las PCTI, advertiríamos que el cambio en el discurso político de algunos gobiernos de la región, como sería el caso de México, pretende apartarse de lo que ha denominado bajo el concepto de "ciencia neoliberal" y que ha sido sustituido en el discurso político gubernamental por el de "ciencia para el pueblo". Este cambio explícito de concepción no revela necesariamente una estrategia reflexiva y novedosa que dé pauta a una nueva concepción de PCTI acorde con nuestras sociedades y sus necesidades sociales, sino un rechazo a lo que se ha hecho con anterioridad, con la consecuente ideologización de las políticas de ciencia y tecnología.

Una nueva concepción en las políticas, sostenida en la idea de que ciencia y tecnología son recursos fundamentales para el logro de objetivos de desarrollo sostenible y de inclusión social, implicaría que la gestión y toma de decisiones sobre el rumbo de esas actividades se sustente en procesos sociales y políticos democráticos. Hay escasos avances en la región en la transición de políticas gubernamentales a políticas públicas que expresen las acciones resultantes de un entramado institucional en el que participan un conjunto de actores, públicos, privados y sociales en torno a un problema público, representado en este caso por la ciencia y la tecnología y su papel en el desarrollo sostenible e incluyente.

Frente a las tendencias que se han instituido para evaluar los avances de los países en su desarrollo científico y tecnológico, en las que se enfatizan indicadores como gasto e inversión, número de investigadores, número de publicaciones en revistas indexadas, medidas de excelencia establecidas en los índices internacionales, entre otros, en una nueva concepción de PCTI se deberá priorizar estrategias de coordinación entre el conjunto de actores que conforman lo que se ha denominado "sistema de ciencia, 
tecnología" que incluye no solamente a las investigadoras e investigadores, las instituciones de educación superior y los centros públicos de investigación en los que se genera el conocimiento, sino también a otros actores que requieren de esas actividades para resolver sus problemas, como los sectores productivos tanto públicos como privados, los sectores sociales y el conjunto de políticas públicas que necesariamente deben sustentarse en conocimiento para la toma de decisiones.

En el plano académico se han generado avances en este sentido que se sustentan en la investigación empírica, a partir de la cual se han documentado procesos que se construyen de abajo hacia arriba y se han conceptualizado, como las redes de conocimiento y espacios regionales y locales de conocimiento (Casas, 2001; Casas, 2003), tecnologías sociales (Dagnino, 2009) y los sistemas tecnológicos sociales (Thomas, 2011). A partir de estas experiencias resulta claro que, para construir una relación directa entre ciencia, tecnología y sociedad, la toma de decisiones, el diseño de políticas y la asignación de recursos deberá sustentarse en procesos participativos de las comunidades científica, académica, tecnológica, de los sectores productivos y sociales y de perspectivas regionales y locales, considerando los intereses y demandas de la sociedad para el mejoramiento de sus condiciones de vida.

En el caso de México, durante las últimas dos décadas y bajo gobiernos con estrategias de desarrollo neoliberal, se avanzó en el diseño institucional de las PCTI, en un conjunto de aspectos relevantes: el marco legal; el tipo de organismos públicos que se han creado y el lugar que ocupan en el aparato gubernamental, los procesos participativos y deliberativos y su importancia para la definición de políticas públicas, la integración horizontal con otras políticas y entidades gubernamentales y el alcance nacional, regional y local de las políticas. En el plano jurídico, a través de la Ley de Ciencia y Tecnología vigente publicada en 2002, reformada en numerosas ocasiones desde esa fecha, se ha puesto en operación un conjunto de instancias orientadas a la coordinación, integración, construcción de procesos interactivos entre actores e instituciones y la descentralización de las capacidades con el propósito de sumar los esfuerzos y orientarlos a sectores específicos. La inclusión en la Ley de Ciencia y Tecnología del Consejo General de Investigación Científica, Desarrollo Tecnológico e Innovación (Artículo 5) del Foro Consultivo Científico y Tecnológico (Artículos 10, 36, 37, 38) como medio de expresión de la comunidad científica, académica, tecnológica y de los sectores productivos, la Red Nacional de Grupos y Centros de Investigación (Artículo 30), la Conferencia Nacional de Ciencia, Tecnología e Innovación 
(Artículo 31) y el establecimiento de convenios con los gobiernos de las entidades federativas y las Secretarías de Estado (Artículo 33) han perseguido ese propósito que debe ser evaluado y en su caso fortalecido.

El andamiaje institucional que se ha ido construyendo a lo largo de 17 años desde la publicación de esta Ley ha enfatizado y puesto en práctica instrumentos de política encaminados a la coordinación del sistema, al fortalecimiento de procesos participativos que ayuden a superar etapas en donde la autoridad presidencial o la de los directivos de Conacyt eran las que guiaban la PCTI (Casas, Corona, Jaso y Vera-Cruz, 2013). La evaluación de sus resultados y la identificación de los procesos de aprendizaje institucional y en particular el diagnóstico de lo acontecido, que son elementos sustantivos en los ejercicios del diseño de políticas públicas, deberían sustentar su reformulación y reorientación para que México pueda disponer de verdaderas PCTI, que equivalen a conjugar intereses de diferentes actores en atención al bien común y al interés público. A 50 años de políticas institucionalizadas en ciencia y tecnología en el país, tenemos que pasar de la improvisación a la profesionalización identificando logros, limitaciones y retos frente a nuevas orientaciones para estas actividades. La idea de un cambio de régimen y de una nueva política para enfrentar el dispendio y la corrupción, lo que sin duda ha minado los esfuerzos realizados durante tantas décadas en la gestión pública, no debe omitir dar cuenta de los aprendizajes institucionales de las políticas, el capital social construido en las entidades federativas y las regiones, los procesos generados de abajo hacia arriba que, independientemente de las políticas, se promueven por la interacción de distintos actores y que representan capacidades interactivas invaluables para construir nuevas políticas públicas (Giraldo, 2019).

En el ejercicio de diseñar las PCTI hay muchas tensiones que no se han resuelto, no solo en México sino en nuestra región, siendo una de las principales las posiciones encontradas de lo que se debe esperar de dichas políticas: un apoyo incremental de recursos para Ciencia, Tecnología e Innovación (CTI) frente a una orientación de esas actividades hacia fines y prioridades específicas. Si esas tensiones no se abordan y se trabajan desde el Estado de manera explícita para encontrar formas de darles cauce, el escenario de confrontación que se vislumbra para el futuro, entre élites y concepciones radicalizadas de ciencia y tecnología, limitará las posibilidades de avanzar como país hacia nuevos paradigmas de política. En un extremo, se promoverá la continuidad a la concepción tradicional y lineal de apoyos progresivos a la ciencia con la idea de que en el largo 
plazo eso contribuirá a resolver los problemas del país, posición que en mi opinión obedece a una mal entendida "libertad de investigación" que fue impulsada a nivel internacional después de la Segunda Guerra Mundial, siguiendo la orientación política que planteó el Informe Valdemar Bush (1945) para contrarrestar los usos militares de la ciencia y que definió el modelo de política de CTI que dominó en el mundo por mucho tiempo y que prevalece aún en la región latinoamericana. Y en el otro extremo se luchará por apoyar una concepción, poco clara aún, que sostiene que la ciencia y la tecnología deben estar orientadas prioritariamente a resolver los problemas de los pobres, de las poblaciones marginadas y en la que se cuestiona cualquier interés económico que permee el desarrollo de la investigación, lo que refleja una posición ideologizada sostenida en visiones radicales sobre los rumbos e impactos de la llamada tecnociencia.

Los gobiernos que se construyen sobre auténticos procesos democráticos han instituido desde hace muchas décadas la creación de organismos para generar procesos participativos y consensos en la toma de decisiones sobre ciencia y tecnología que contemplen los usos sociales de estas actividades y su apropiación social. Se trata de procesos sociales que implican nuevas formas de generación de conocimiento en interacción con los actores que demandan de estos recursos para resolver problemas o definir políticas públicas. La apropiación involucra nuevos modelos de participación pública en la ciencia y nuevos modelos de concebir la relación entre ciencia, tecnología y sociedad y de formular las políticas de CTl; implica la co-producción de conocimientos, el fortalecimiento de las capacidades colectivas para usar los conocimientos y tomar decisiones conociendo sus beneficios y riesgos. Transitar a esas nuevas formas implicaría fortalecer las instancias institucionales que fomenten la participación.

En México estamos frente a una oportunidad de redirigir la política de CTI en nuestro país, vincularnos a los esfuerzos innovadores de políticas que se realizan en otras partes del mundo y de nuestra región latinoamericana orientados a la inclusión social, pero también nos encontramos ante el riesgo que la falta de experiencia en el diseño de políticas públicas, la radicalización de posiciones políticas y la lucha entre grupos de interés por el control de los recursos, nos lleve, no solo a seguir construyendo capacidades de ciencia y tecnología cada vez más desvinculadas de la sociedad, sino a destruir las escasos y precarios eslabones existentes entre ciencia y sociedad. 


\section{Referencias bibliográficas}

1. ARANCIBIA, Eliana Alejandra. (2019). Ciencia, tecnología e innovación y políticas para la inclusión social en América Latina (pp.08-35). En: CASAS, Rosalba (comp.), Conocimiento y procesos interactivos en contextos territoriales. Nuevas dimensiones en el análisis de las políticas de ciencia y tecnología (libro en dictamen).

2. BUSH, Valdemar. (1945). Science: The Endless Frontier. North Stratford: ACLS Humanities E-Book. 248 pp.

3. CASAS, Rosalba. (2001). La formación de redes de conocimiento. Barcelona: Anthropos, IIS-UNAM. 380 pp.

4. CASAS, Rosalba. (2003). Intercambio de flujos de conocimiento en las redes (pp. 306-354) En: LUNA, M. (coord.), Itinerarios del conocimiento; Formas dinámicas y contenido, un enfoque de redes. Barcelona: Anthropos, IIS-UNAM.

5. CASAS, Rosalba, CORONA Juan Manuel, y RIVERA, Roxana. (2014). Políticas de Ciencia, Tecnología e Innovación en América Latina: entre la competitividad y la inclusión social (pp. 352-264). En: KREIMER, Pablo, VESSURI, Hebe y ARELLANO, Lea (ed.), Perspectivas Latinoamericanas en el Estudio Social de la Ciencia, la Tecnología y el Conocimiento. Ciudad de México: Red Cyted/FCCyT, Siglo XXI.

6. CASAS, Rosalba, CORONA Juan Manuel, JASO, Marco y VERA-CRUZ, Alexandre. (2013). Construyendo el Diálogo entre los Actores del Sistema de Ciencia, Tecnología e Innovación. Libro conmemorativo de los 10 años del Foro Consultivo Científico y Tecnológico. Ciudad de México: Gustavo Casasola. 191 pp.

7. DAGNINO, Renato. (2009). Tecnologia Social. Ferramenta para construir outra sociedade. $1^{\text {a }}$ ed. Campinas: Editora Kaco. 95 pp.

8. GIRALDO, María Elena. (2019). Políticas Regionales de Ciencia y Tecnología: capacidades interactivas, redes y desarrollo territorial en dos parques tecnocientíficos de México y Colombia. Ciudad de México: Centro de Investigaciones sobre América Latina y el Caribe (CIALC), UNAM. 414 pp.

9. MÉXICO. Congreso General de la Unión. (2002). Ley de Ciencia y Tecnología. Nueva Ley publicada en el Diario Oficial de la Federación el 5 de junio de 2002.

10. THOMAS, Hernán. (2011). Tecnologías sociales y ciudadanía socio-técnica. Notas para la construcción de la matriz material de un futuro viable. Revist@ do Observatório do Movimento pela Tecnologia Social da América Latina Ciência \& Tecnologia Sociedade, $N^{\circ} 1$, Pp.1-22.

11. VELHO, Lea. (2011). La ciencia y los paradigmas de la política científica, tecnológica y de innovación (pp. 99-125). En: ARELLANO, Lea y KREIMER, Pablo (dir.), Estudio Social de la Ciencia y la Tecnología desde América Latina. Bogotá: Siglo del Hombre Editores.

Para citar este artículo:
CASAS, Rosalba. (2020). Políticas públicas de ciencia y tecnología en América Latina. Ante la encrucijada de los cambios políticos. Teuken Bidikay, Vol. 11, N 16. Medellín: Politécnico Colombiano. Pp. 21-28. doi: 10.33571/teuken.v11n16a1 